\title{
The Non-Smooth Pitchfork Bifurcation: A Renormalization Analysis
}

\author{
L N C Adamson*and A H Osbaldestin ${ }^{\dagger}$ \\ Department of Mathematics \\ University of Portsmouth \\ Portsmouth \\ PO1 3HF, UK
}

January 7, 2015

\begin{abstract}
We give a renormalization group analysis of a system exhibiting a non-smooth pitchfork bifurcation to a strange non-chaotic attractor. For parameter choices satisfying two specified conditions, self-similar behaviour of the attractor on and near the bifurcation curve can be observed, which corresponds to a periodic orbit of an underlying renormalization operator. We examine the scaling properties for various parameter choices including the so-called Pitchfork Critical Point. Finally, we study the autocorrelation function for the system and show that it is equivalent to that present in symmetric barrier billiards.
\end{abstract}

\section{Introduction}

Strange non-chaotic attractors (SNAs) have been the focus of a broad wave a research into quasiperiodically forced systems over the past three decades. This began when Grebogi et al. (GOPY) gave an analytical proof of their existence in [8]. These objects are seemingly paradoxical in nature; in essence we have an attracting set which is not finite and which is nowhere differentiable (fractal in fact), and yet nearby orbits on it do not diverge at an exponential rate over time. Indeed, prior to their discovery the terms 'strange' and 'chaotic' were used like synonyms.

These pioneering authors then extended their work into to the evolution of these attractors in [3], where for a generalised circle map the transition between quasiperiodic, strange non-chaotic and chaotic behaviour was studied. For the map under study there were three regions in two-dimensional parameter space which gave rise to these behaviours. It was shown that the set in parameter space for which the system exhibits SNAs has Cantor set-like structure and lies between two boundary curves separating the other two behaviours, thus making it an intermediate between quasi-periodic and chaotic motion. In [16] it is shown that these attractors occur on a set of positive measure, thus making them physically important.

Further work on the characterisation of SNAs was presented in [15]. This gave a number of conditions which enable one to determine whether the attracting set is strange, which is highly useful as this is often difficult to determine analytically.

Of interest to us in this paper is the model studied by Glendinning in [7], in which the non-smooth pitchfork bifurcation for SNAs is studied. The results of this paper will be summarised for a qualitatively equivalent model in

*luke.adamson@port.ac.uk

†andrew.osbaldestin@port.ac.uk 
Section 2. The aim of this paper is to provide a renormalization group approach along the entire curve in parameter space on which this bifurcation occurs.

Furthermore, in [10] a renormalization group approach was developed to study a route to SNA labelled the "blowout birth". In fact, this is just the non-smooth pitchfork bifurcation studied by Glendinning but for a particular choice of parameter. In [10] Kuznetsov et al. demonstrated that for a model qualitatively similar to that studied in [8], there are local scaling properties for certain choices of initial phase at the critical point in parameter space which separates a trivial attractor from SNAs. Furthermore, it is demonstrated that these scaling properties can be used to examine the size and structure of the SNA near the critical point, demonstrating the self-similar structures which occur on smaller and smaller scales, and thus giving a full understanding of this bifurcation.

Work into the correlations and spectra of SNAs has been presented in [14], showing that they exhibit unusual properties. The autocorrelation function $(\mathrm{ACF})$ is self-similar with peaks occurring at resonant frequencies with respect to the forcing, and the spectrum is a fractal curve on the complex plane. A renormalization approach was developed to study the ACF of the GOPY model in [6]. Numerically it was observed in [6] that the self-similarity could be understood in terms of a period six orbit of a renormalization operator.

This topic was then rigorously examined in [11], showing that the autocorrelation function has peaks of magnitude $1-1 / \sqrt{5} \simeq 0.55 \ldots$ at every third Fibonacci number for the golden mean forcing frequency, putting on firmer footing earlier numerical results in [14] and [6]. This will be extended for the model under study in this paper in Section 4.

We begin with a review of the features of Glendinning's model [7], but for which we consider a different, but qualitatively equivalent variant. In Section 3 we develop the renormalization group approach similar to that in [10] and explain the similarity between this and the more general approach seen for similar models (a thorough summary can be seen in [5]). We give necessary conditions on parameters, scaling factors and initial phases for periodic behaviour of the renormalization operator to occur, in addition to explicit construction of these periodic orbits using a method of numerical approximation based on analysis of the growth rate of the attractor. Finally in Section 4 we provide a link between the ACF of the system under study and the ACF which appears in the study of work we have previously conducted on symmetric barrier billiards [13].

\section{The map and previous work}

The map of interest to us in this paper is a generalisation of the map studied in [10] for which a renormalization group approach was used to give an analysis of the birth of an SNA. It is given by

$$
\begin{aligned}
& x_{i+1}=f\left(\theta_{i}, x_{i}\right)=2 \sigma\left(\phi+\cos \left(2 \pi \theta_{i}\right)\right) \underbrace{\frac{x_{i}}{\left(1+x_{i}^{2}\right)^{1 / 2}}}_{*}, \\
& \theta_{i+1}=\theta_{i}+\omega \quad(\bmod 1) .
\end{aligned}
$$

For simplicity in the renormalization analysis, we take $\omega$ to be the inverse of the golden mean throughout i.e. $\omega=(\sqrt{5}-1) / 2$, although generalization to a broader class of quadratic irrationals is possible [1]. The nonlinear function * highlighted in (2.1) is qualitatively equivalent to $\tanh (x)$, and in [7] this system was studied by Glendinning for the latter choice. The reason for the change to form * is the same as it was in [10] where the same model (with cos replaced by $\sin$ ) was studied for the case of $\phi=0$; composition of functions in this class yield another function in this class, which means that in the renormalization analysis the $x$ variable at characteristic times will also be a member of this class. In particular if $f_{1,2}(x)=A_{1,2} x\left(1+B_{1,2} x^{2}\right)^{-1 / 2}$ then $\left.f_{1}\left(f_{2}(x)\right)=A_{1} A_{2} x\left(1+\left(B_{2}+B_{1} A_{2}^{2}\right) x^{2}\right)\right)^{-1 / 2}$.

Due to the qualitative similarity of the system (2.1) - (2.2) to that studied by Glendinning, the arguments in [7] can be carried over directly and will now be summarised. 


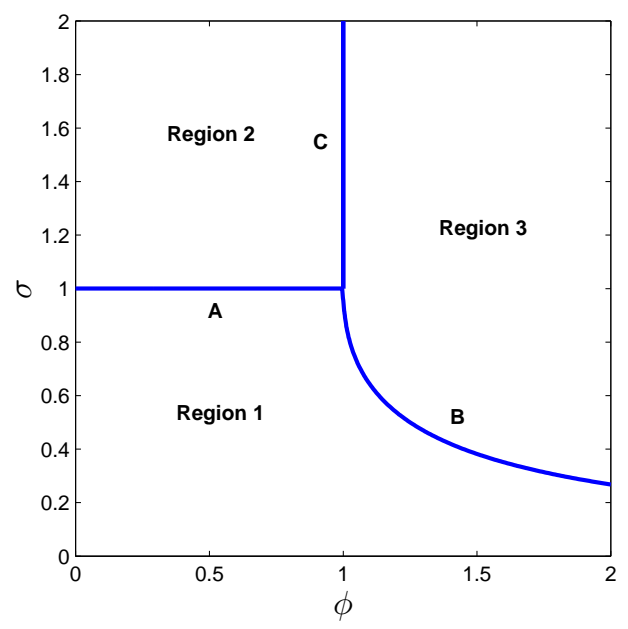

Figure 1: Plot of the first quadrant of the parameter plane $(\phi, \sigma)$ showing the three regions in which different dynamical regimes occur and the bifurcation curves $A, B$ and $C$ which separate them.

The circle in phase space defined by

$$
\mathcal{L}=\{(\theta, x) \mid x=0\},
$$

is invariant under the map, and the stability of this set can be determined by calculation of its transverse Lyapunov exponent. Using the ergodicity in $\theta$ we can write this exponent as

$$
\begin{aligned}
\lambda & =\int_{0}^{1} \ln \left|\frac{\partial f}{\partial x}\right|_{x=0} \mathrm{~d} \theta, \\
& =\frac{1}{2} \int_{0}^{1} \ln (2 \sigma(\phi+\cos (2 \pi \theta)))^{2} \mathrm{~d} \theta .
\end{aligned}
$$

In [7] it is shown that this integral is given by

$$
\lambda= \begin{cases}\ln \sigma, & 0 \leq \phi \leq 1, \\ \ln \sigma+\ln \left(\phi+\sqrt{\phi^{2}-1}\right), & \phi>1 .\end{cases}
$$

As shown in Figure 1, (2.6) defines two bifurcation curves $A$ and $B$ on which the Lyapunov exponent for $\mathcal{L}$ is zero. In particular these curves are given by

$$
\begin{aligned}
& A=\{(\phi, \sigma) \mid \sigma=1,0 \leq \phi \leq 1\}, \\
& B=\left\{(\phi, \sigma) \mid \sigma=\left(\phi+\sqrt{\phi^{2}-1}\right)^{-1}, \phi>1\right\} .
\end{aligned}
$$

The circle $\mathcal{L}$ is thus stable for parameter choices below the union of these curves, and unstable above them. However, one more bifurcation takes place, as the system (2.1)-(2.2) becomes invertible for $\phi>1$. The behaviour below the union of curves $A$ and $B$ (Region 1 in Figure 1) is trivial as the attractor is simply $\mathcal{L}$ as shown in [9]. Hence we define a third bifurcation curve as

$$
C=\{(\phi, \sigma) \mid \phi=1, \sigma \geq 1\} .
$$

We now focus on the dynamics in Regions 2 and 3 shown in Figure 1. For choices of parameter in Region 2 it is shown in [7] (following the approach in [8]) that the system gives rise to a strange non-chaotic attractor (SNA). In this region the set $\mathcal{L}$ is unstable and because $\phi<1$ there are values $\tilde{\theta}$ such that $\phi+\cos (2 \pi \tilde{\theta})=0$. Now, as $x=0$ is invariant we conclude that the set of points $(\tilde{\theta}+n \omega, 0)$ are all members of the attracting set for $n \in \mathbb{N}$. Thus there is a dense set of points on the $\theta$ axis such that $x=0$. However as $\mathcal{L}$ is unstable it is not the attractor and so there are non-zero values of $x$. This leads to a pinching effect indicating that the resulting attractor is not differentiable at any point and is thus strange. 
To prove that the attractor is non-chaotic for any choice of parameter not on one of the bifurcation curves $A$ and $B$, we use the approach shown in [8] to prove that the Lyapunov exponent is non-positive. Firstly we note that

$$
\frac{\mathrm{d}}{\mathrm{d} x}\left(\frac{x}{\sqrt{1+x^{2}}}\right) \leq \frac{1}{\sqrt{1+x^{2}}},
$$

with equality only being true at $x=0$. Inside Region 1 the attractor is $\mathcal{L}$, so by our calculation of the transverse Lyapunov exponent for $\mathcal{L}$ above we conclude that the exponent is negative for any parameter choice inside Region 1. In Regions 2 and $3 x$ does not tend to zero and so the inequality is again strict. Hence

$$
\left|\frac{\partial f}{\partial x}\right|_{x_{i}, \theta_{i}}<\left|\frac{x_{i+1}}{x_{i}}\right|
$$

and so from the definition of the Lyapunov exponent we have

$$
\lambda=\lim _{N \rightarrow \infty}\left(\frac{1}{N} \sum_{i=1}^{N} \ln \left|\frac{\partial f}{\partial x}\right|_{x_{i}, \theta_{i}}\right) \leq \lim _{N \rightarrow \infty}\left(\frac{1}{N} \sum_{i=1}^{N} \ln \left|\frac{x_{i+1}}{x_{i}}\right|\right)=\lim _{N \rightarrow \infty}\left(\frac{1}{N} \sum_{i=1}^{N} \ln \left|x_{i+1}\right|-\ln \left|x_{i}\right|\right)=0 .
$$

In Figure 2a we show a plot of the SNA occurring for parameter choice $(\phi, \sigma)=(0.5,1.5)$ inside Region 2 . The numerically calculated Lyapunov exponent in this case is -0.811 .

In Region 3 when $\phi>1$ the map becomes invertible as the factor $\phi+\cos (2 \pi \theta)$ is never equal to zero, and thus the "pinching" effect which occurs for Region 2 is absent and we get a smooth attracting torus as demonstrated in [7]. The set $\mathcal{L}$ is unstable. Due to the $x \rightarrow-x$ symmetry in the underlying map there is in fact a pair of symmetric attractors, one above and the other below $\mathcal{L}$. In Figure $2 \mathrm{~b}$ we give a plot of an attractor in this region. The numerically calculated Lyapunov exponent is -2.736 .

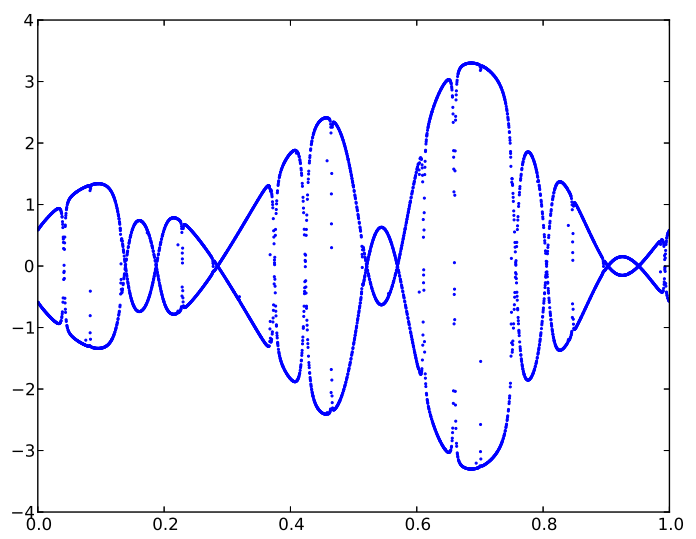

(a) Strange non-chaotic attractor in Region 2 for parameter choice $(\phi, \sigma)=(0.5,1.5)$.

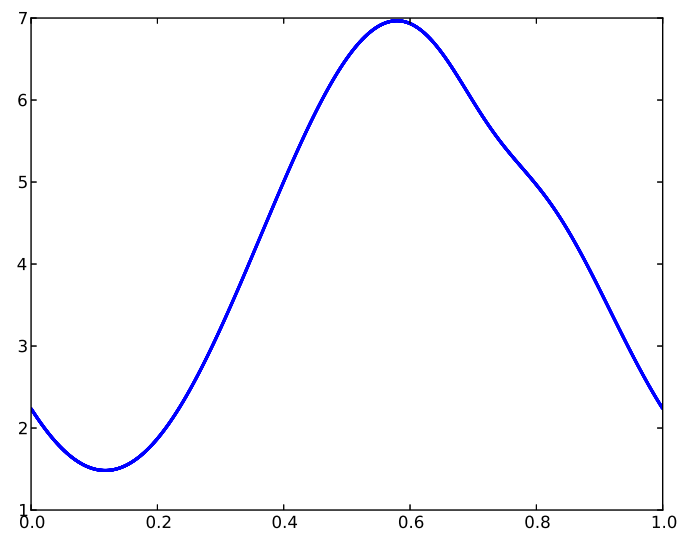

(b) Torus attractor in Region 3 for parameter choice $(\phi, \sigma)=(1.5,1.5)$.

Figure 2: Plots demonstrating the different asymptotic behaviours of system (2.1)-(2.2) in Regions 2 and 3.

We consider the effect of the overall bifurcation as in [7]. Consider any simple path in parameter space passing through bifurcation curve $B$ (but not the co-dimension two point $(1,1)$ ). The effect of this bifurcation is that the stable circle $\mathcal{L}$ loses stability and a pair (considering symmetry) of stable invariant curves is created. This is the effect of the standard pitchfork bifurcation for curves.

Now consider a path crossing bifurcation curve $A$ (again excluding $(1,1)$ ). In this case $\mathcal{L}$ becomes unstable and the pinching effect gives rise to an SNA. This attractor is bounded by two stable symmetric semi-continuous (but nowhere differentiable) curves $\pm \zeta(x)[7]$, and can thus be considered as a "non-smooth pitchfork bifurcation". 
Finally consider the effect if the path crosses $C \backslash(1,1)$; the pinching effect no longer occurs and $\mathcal{L}$ remains unstable, and two symmetric stable invariant curves are created (which near $C$ will tend towards $\pm \zeta(x)$ ). Here the attractor is annihilated to give two attracting sets and one unstable set, and is thus another form of pitchfork bifurcation.

\section{Renormalization analysis}

In the main body of this article we analyse the scaling properties of the attractors for choices of parameter on the bifurcation curve $A$ and in particular to the co-dimension 2 point $(1,1)$, which is the meeting point of all three curves and thus separates three regimes of dynamical behaviour. Our approach will be similar to that in [10] where a renomalization analysis of the "birth of a strange non-chaotic attractor" was given.

To begin the analysis we separate the system into two component parts which reflect the different behaviours of the system. In particular we have an oscillating component $\mathcal{O}_{i}$ and an amplitude component $\mathcal{A}_{i}$ such that $x_{i}=\mathcal{A}_{i} \mathcal{O}_{i}$ where

$$
\begin{aligned}
& \mathcal{O}_{i+1}=2\left(\phi+\cos \left(2 \pi \theta_{i}\right)\right) \mathcal{O}_{i}, \quad \mathcal{O}_{0}=1, \\
& \mathcal{A}_{i+1}=\sigma \frac{\mathcal{A}_{i}}{\left(1+\mathcal{O}_{i}^{2} \mathcal{A}_{i}^{2}\right)^{1 / 2}}, \quad \mathcal{A}_{0}=x_{0} .
\end{aligned}
$$

As $\omega$ is the inverse of the golden mean, it makes sense to study the system at the Fibonacci times $F_{n}$, which satisfy the recurrence $F_{n+1}=F_{n}+F_{n-1}, F_{0}=0, F_{1}=1$, and are the continued fraction convergents to $\omega$. This analysis can be generalized to a class of quadratic irrational frequencies as was achieved in [1] for symmetric barrier billiards.

We will assume from now on that $\sigma=1$ as we are interested in bifurcation curve $A$ which exhibits the non-smooth pitchfork bifurcation. Due to the aforementioned invariance of the expression underbraced in (2.1), we can now write

$$
\begin{aligned}
\mathcal{O}_{F_{n}} & =T_{n}(\theta), \\
\mathcal{A}_{F_{n}} & =\frac{\mathcal{A}_{0}}{\left(1+U_{n}(\theta) \mathcal{A}_{0}^{2}\right)^{1 / 2}},
\end{aligned}
$$

where

$$
\begin{aligned}
T_{n}(\theta) & =\prod_{i=0}^{F_{n}-1} 2(\phi+\cos (2 \pi(\theta+i \omega))), \\
U_{n}(\theta) & =\sum_{i=0}^{F_{n}-1} \mathcal{O}_{i}^{2} .
\end{aligned}
$$

Note that we are expressing the initial phase without subscript as $\theta$ to highlight the fact that we are dealing with functions of this variable. Using the Fibonacci recurrence, we deduce that

$$
\begin{aligned}
& T_{n+2}(\theta)=T_{n+1}(\theta) T_{n}\left(\theta-(-\omega)^{n+1}\right), \\
& U_{n+2}(\theta)=U_{n+1}(\theta)+T_{n+1}^{2}(\theta) U_{n}\left(\theta-(-\omega)^{n+1}\right) .
\end{aligned}
$$

Here we have made use of the identity

$$
F_{n+1} \omega=F_{n}-(-\omega)^{n+1}
$$

which implies that $\theta_{F_{n+1}}=\theta-(-\omega)^{n+1} \bmod 1$. As a consequence of this result, we see that the characteristic scale in $\theta$ per iteration is $(-\omega)$. We now let some $\theta^{0}$ be the origin (the initial phase) via the coordinate transformation $u=\theta-\theta^{0}$ (note here how we have relabeled $\theta$ as $u$, which reflects the fact that in the renormalization analysis this variable loses its significance as a phase) and renormalize with respect to $u$ by defining the rescaled functions $Q_{n}(u)=T_{n}\left((-\omega)^{n} u+\theta^{0}\right)$ and $H_{n}(u)=U_{n}\left((-\omega)^{n} u+\theta^{0}\right)$. Using recurrences (3.7)-(3.8) we can derive (as in [10]) recurrences for these functions as

$$
\begin{aligned}
& Q_{n+2}(u)=Q_{n+1}(-\omega u) Q_{n}\left(\omega^{2} u+\omega\right), \\
& H_{n+2}(u)=H_{n+1}(-\omega u)+Q_{n+1}^{2}(-\omega u) H_{n}\left(\omega^{2} u+\omega\right) .
\end{aligned}
$$


The initial conditions for these recurrences are $Q_{0}=1, Q_{1}(u)=T_{1}\left(\theta^{0}-\omega u\right)=2\left(\phi+\cos \left(2 \pi\left(\theta^{0}-\omega u\right)\right)\right)$ and $H_{0}=0$, $H_{1}=1$. Note that this corrects an error in [10], where the change in coordinates was not implemented correctly into the definition of $Q_{n}$ and $H_{n}$. This did not affect the numerical results in that paper, but we have found that it leads to incorrect scaling factors for certain choices of the initial phase.

This system was studied previously for the case $\phi=0$ in [10] for the study of the "birth of an SNA". In [10] it is shown that for certain choices of initial phase, iteration of (3.10) leads to periodic behaviour of $Q_{n}$. Due to the results of previous work in [11] (for example), we conclude that a necessary condition for periodicity is that the zeros of the initial condition $Q_{1}$ lie in $\mathbb{Q}(\omega)$, the field of rationals over $\omega$.

In this case we see that (3.11) is a periodically driven linear recurrence (although the linearity is deceptive!). Assuming the period of $Q_{n}^{2}$ is $p$, we expect it to produce a factor of growth over a period i.e. $H_{n+p}(u) \simeq \nu^{2} H_{n}(u)$. Due to the fact that $U_{n}$ describes the growth of the attractor, and due to the form of (3.2), we conclude that the amplitude of the attractor decreases by a factor of $\nu$ over a period. This approach enables us to see how the constituent parts of the underlying system behave at smaller and smaller scales.

We remark that in keeping with approaches used elsewhere (for extensive examples see [5]) we could have formulated this problem slightly differently. In particular we define the functions $f_{n}(x, \theta)$ by the equation

$$
x_{i+F_{n}}=f_{n}\left(x_{i}, \theta_{i}\right) .
$$

The apparent similarity between these two methods is seen as we can now write

$$
f_{n}(x, \theta)=\frac{T_{n}(\theta) x}{\sqrt{1+U_{n}(\theta) x^{2}}} .
$$

Indeed, up until this point both approaches are identical. Once again we define a new coordinate system $u=\theta-\theta^{0}$ and we now renormalize by changing the scales of $x$ and $u$ by defining the rescaled functions

$$
g_{n}(x, u)=\alpha^{n} f_{n}\left(x / \alpha^{n},(-\omega)^{n} u+\theta^{0}\right) .
$$

The scale in $u$ is the same as before (for $\theta$ ) but the new variable $\alpha$ represents the change in scale for $x$ per step of the renormalization. By manipulating (3.14) the renornalization operator can be shown to be given by

$$
g_{n+2}(x, u)=\alpha^{2} g_{n}\left(\alpha^{-1} g_{n+1}\left(x / \alpha,-u \omega+(-\omega)^{-(n+1)} \theta^{0}\right), \omega^{2} u+\omega+(-\omega)^{-n} \theta^{0}\right) .
$$

For the model under study, direct substitution yields

$$
g_{n}(x, u)=\frac{Q_{n}(u) x}{\sqrt{1+\tilde{H}_{n}(u) x^{2}}},
$$

where $\tilde{H}_{n}(u)=\alpha^{-2 n} H_{n}(u)$, and $Q_{n}, H_{n}$ are as defined in (3.10)-(3.11). Hence the difference is in the scaling of $H_{n}$. This is a correction of the previous approach in [5], and we will now clarify the relationship between the two renormalization schemes. Indeed, we can now pick $\alpha$ to ensure periodicity (with period $p$ ) of $\tilde{H}$. Asymptotically we have

$$
\alpha^{2(n+p)} \tilde{H}_{n+p}(u)=H_{n+p}(u)=\nu^{2} H_{n}(u)=\nu^{2} \alpha^{2 n} \tilde{H}_{n}(u) .
$$

Thus for periodicity we require $\alpha=\nu^{1 / p}$.

We will now determine conditions on $\phi$ and $\theta^{0}$ necessary for periodic behaviour to occur. In particular, the zeros of $Q_{1}$ are given by

$$
x=\omega^{-1}\left( \pm \frac{\cos ^{-1}(-\phi)}{2 \pi}+\theta^{0}+n\right), \quad n \in \mathbb{Z} .
$$

The conditions for these points to be in $\mathbb{Q}(\omega)$ are clearly that $\theta^{0}+\cos ^{-1}(-\phi) / 2 \pi \in \mathbb{Q}(\omega)$ and $\theta^{0}-\cos ^{-1}(-\phi) / 2 \pi \in$ $\mathbb{Q}(\omega)$ (note that $\left.\omega^{-1}=1+\omega\right)$. Hence we require that both $\theta^{0}$ and $\cos ^{-1}(-\phi) / \pi$ are in $\mathbb{Q}(\omega)$.

For each value of $\phi$ satisfying this constraint, we can pick any $\theta^{0} \in \mathbb{Q}(\omega)$ as an origin, and we expect that each choice will give different local scaling properties due to the multi-fractal nature of the SNA [6]. The case $\phi=0$ was 


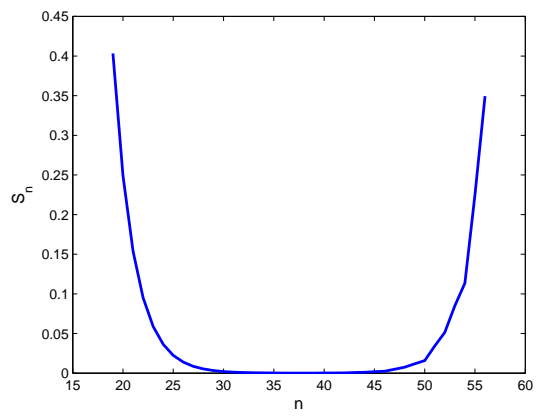

Figure 3: Plot of the standard deviation $S_{n}$ of $\gamma_{n}$ at time index $n$ for $n=19, \ldots, 57\left(\phi=0.5, \theta^{0}=0\right)$.

studied extensively in [10] for several initial phases $\theta^{0}$ in $\mathbb{Q}(\omega)$, each of which resulted in periodic behaviour of the renormalization operator as our theory predicts. We will now illustrate the material derived thus far in this section by examining the case of $\phi=0.5$. In this case $\cos ^{-1}(-\phi) / \pi=2 / 3$ and so with a choice of $\theta^{0}$ in $\mathbb{Q}(\omega)$ we expect periodicity of (3.10) and therefore a scaling law in a vicinity of $\theta^{0}$.

We take $\theta^{0}=0$, and represent the resulting $Q_{1}$ by a polynomial interpolant accurate to machine precision. This is achieved using the Chebfun system, an incredibly useful extension developed for Matlab. See (for example) [4] for more details. Iterating forward we calculate that $Q_{n}^{2}$ converges to a period four orbit. Due to the chaotic nature of the inverse of the iterated function system given by the contractions $\phi_{1}(x)=-\omega x$ and $\phi_{2}(x)=\omega^{2} x+\omega$, after a finite number of iterations this periodicity is no longer observable as the system degenerates to noise. Thus we have a finite range of iterates for which the initial transient behaviour has disappeared and in which the system has not degenerated to noise, leaving us with the periodic orbit we desire.

We can estimate $\nu$ by looking at the periodic growth functions given by

$$
\gamma_{n}(u)=\frac{H_{n+p}(u)}{H_{n}(u)}
$$

From (3.17) we expect that asymptotically $\gamma_{n} \rightarrow \nu^{2}$ as $n \rightarrow \infty(p=4)$. By substituting the periodic solution of (3.10) into (3.11) we examine the standard deviation of $\gamma_{n}(u), S_{n}$. Due to the previous argument with regard to the (numerical) evolution of $Q_{n}$, there will be transient behaviour followed by periodicity which will then degenerate to noise. Hence a plot of $S_{n}$ against $n$ will produce a "U-shaped" curve; the flat region is indicative of convergence to the desired periodic orbit, whereas the left and right sides correspond to transients and noise respectively. This is shown in Figure 3 for the case under study. We choose the $n$ which minimises the standard deviation, and thus the $\gamma_{n}$ which is the "most constant". Then we calculate the mean value of $\gamma_{n}$ and take the square root of it to obtain $\nu$.

We have checked the proposed method of estimating $\nu$ by comparing the factors to those obtained in [10] for $\phi=0$ with cos replaced by $\sin$ in (2.1). For example in the case $\theta^{0}=0.25$ we find the scaling factor to four decimal places to be $\nu=7.4246$, in perfect agreement with [10].

Returning to the problem under study $\left(\phi=0.5, \theta^{0}=0\right)$, we find $n=37$ to give a minimum standard deviation of $\simeq 7 \times 10^{-5}$, and by calculating the mean value of $\gamma_{37}$ we estimate the value of $\nu$ to be $\nu=4.127$ (to $3 \mathrm{~d}$.p). This gives us the scaling factor for $x$ as $\alpha=\nu^{1 / 4}=1.425$ (again to 3 d.p).

Note that $Q_{n}$ is actually periodic with period eight, but due to a symmetry in the solutions the period of $Q_{n}^{2}$ is four. We can now substitute these solutions into (3.16) and plot the period eight orbit of the renormalization operator $g_{n}(x, u)$ as shown in Figure 4.

Hence the evolution of the system at time $F_{k}$ is the same after appropriate scaling as the system at time $F_{8 m+k}$. In fact however, due to the aforementioned symmetry which can also seen in Figure 4, the system actually displays similar behaviour every four steps of the renormalization operator as the model under study is symmetric. In particular, this means that the dynamics with some starting point $(x, u)$ is the same as the dynamics starting from 

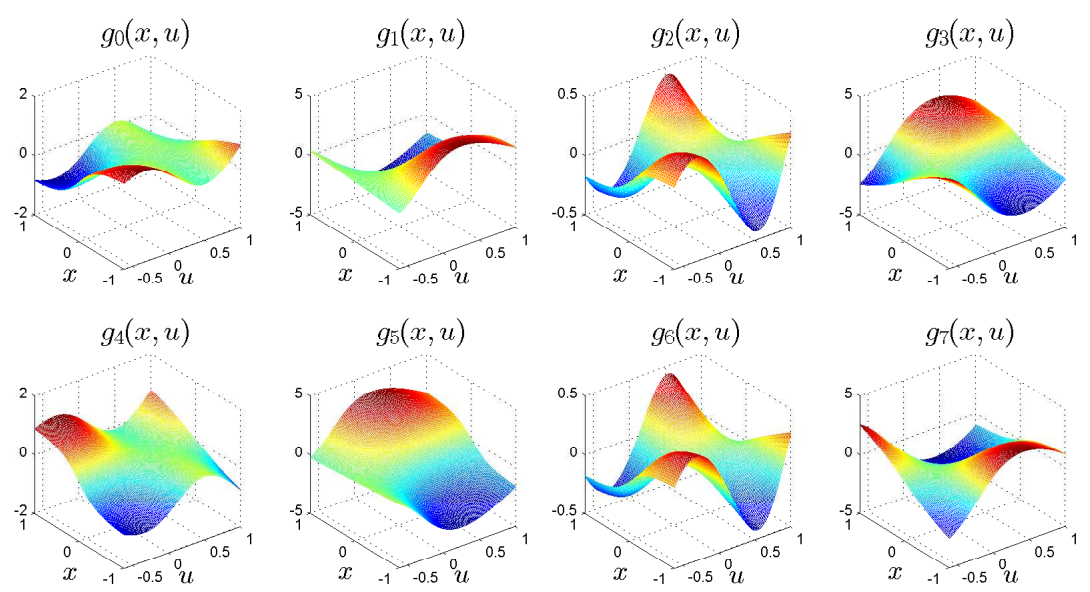

Figure 4: Plot of $g_{n}(x, u)$ showing the period eight orbit of the renormalization operator.

$x / \nu$ and $u /(-\omega)^{4}$ with time scaled by $\omega^{-4}$.

It follows from the fact that $H_{n}$ describes the growth of $U_{n}$ and then from (3.4) that the scaling factor for the amplitude $\mathcal{A}$ is $\nu$. Thus when time is increased by a factor $F_{n+4} / F_{n} \rightarrow \omega^{-4}$ at the critical point $(0.5,1)$ in parameter space, the amplitude of the attractor decreases by the factor $\nu$. Hence the amplitude decays like $i^{-\kappa}$ where $\kappa=-\ln (\nu) / 4 \ln (\omega)=0.736 \ldots$ and $i$ is the time index. A plot of the amplitude on a logarithmic scale is shown in Figure 5, along with a plot of the expected decay (in red) given by $i^{-\kappa}$, which illustrates that the amplitude decays by a constant factor $\nu$ when time is increased by a factor of $\omega^{-4}$. A similar plot is given by Figure 3 in [10] for the case corresponding to $\phi=0$.

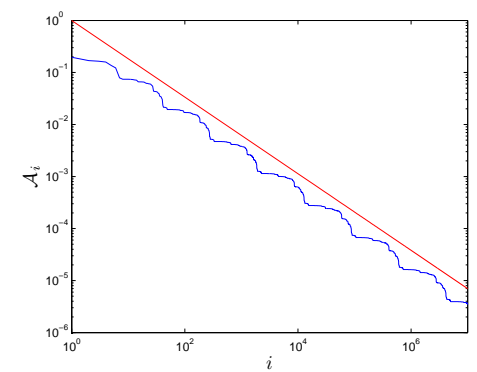

Figure 5: Plot of $\mathcal{A}_{i}$ against time index $i$ on a logarithmic scale, in addition to the decay rate suggested by the renormalization analysis.

Having considered the system at the critical point, we now consider the behaviour of the system near to the critical point. A small perturbation of $\phi$ will mean that $\cos (-\phi) / \pi \notin \mathbb{Q}(\omega)$ and will thus destroy the periodicity of $Q_{n}$ (although for a sufficiently small perturbation the pictures will look similar to those at the critical point). Hence there will be no scaling law in an $\epsilon$ neighbourhood of $\phi$ for $\epsilon$ sufficiently small. For a perturbation in $\sigma$ things are different however. Using the same approach presented in [10] (which was used for the case $\phi=0$ ), near the critical point we can write $\sigma=\exp (\epsilon)$ for some $\epsilon>0$. After $N$ iterations of (2.1) the multiplying factor is $\sigma^{N}=\exp (N \epsilon)$, and so the perturbation $\epsilon$ is renormalized with the same factor as time, $\omega^{-4}$.

To demonstrate the self similarity near the initial phase $\theta^{0}=0$, we first pick a $\delta_{0}$ neighbourhood of $\theta^{0}$ and plot the attractor in this neighbourhood for $\sigma_{0}=\exp \left(\epsilon_{0}\right)=1+\epsilon_{0}+O\left(\epsilon_{0}^{2}\right)$. According to our rescaling law by setting $\sigma_{1}=\exp \left(\omega^{4} \epsilon_{0}\right)=\exp \left(\epsilon_{1}\right)=1+\omega^{4} \epsilon_{0}+O\left(\epsilon_{0}^{2}\right)$, multiplying $x$ by $\nu$, setting $\delta_{1}=\delta_{0} \omega^{4}$ and rescaling $\theta$ by the factor 
$(-\omega)^{-4}$ we should observe a situation similar to the original choice.

Figure 6 shows the rescaled attractors obtained for three such iterations of the above procedure, showing the self-similarity near the critical point.

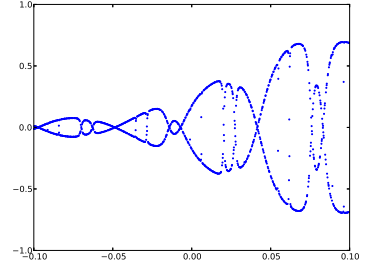

(a) SNA for parameter choice $(\phi, \sigma)=\left(0.5,1+\epsilon_{0}\right)$.

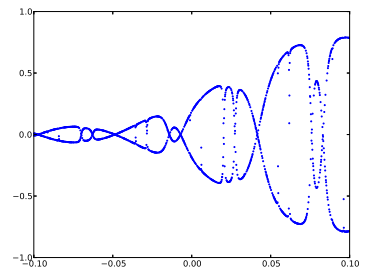

(c) Rescaled strange nonchaotic attractor for parameter choice $(\phi, \sigma)=\left(0.5,1+\omega^{8} \epsilon_{0}\right)$

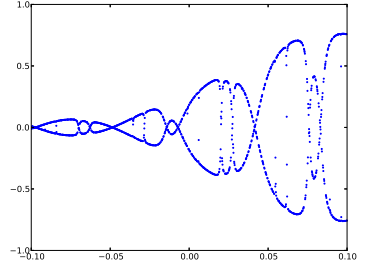

(b) Rescaled strange nonchaotic attractor for parameter choice $(\phi, \sigma)=\left(0.5,1+\omega^{4} \epsilon_{0}\right)$

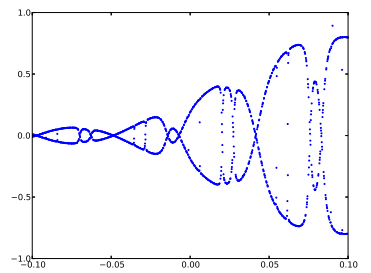

(d) Rescaled strange nonchaotic attractor for parameter choice $(\phi, \sigma)=\left(0.5,1+\omega^{12} \epsilon_{0}\right)$

Figure 6: Plots showing self-similarity near the critical point $(0.5,1)$ with $\epsilon_{0}=\delta_{0}=0.1$.

\subsection{The Pitchfork Critical Point (PCP)}

We now turn our attention to the co-dimension two point in parameter space $(\phi, \sigma)=(1,1)$ which is the intersection point for all three boundary curves $A, B$ and $C$.

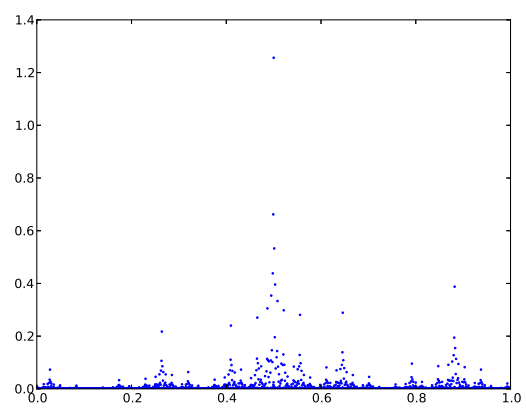

Figure 7: Attractor at the PCP

The fact that this point is surrounded by the three different types of dynamical regime occurring in the system suggests that an understanding of the behaviour at and near this point would be beneficial to the overall understanding 
of this system. The attractor at the PCP is shown in Figure 7.

The numerical methods developed in this section can also be applied to this point, and once again (for simplicity) we take $\theta^{0}=0$. Iterating forward (3.10) we discover $Q_{n}$ converges to a period three orbit, and so to does $Q_{n}^{2}$. Working to 3 decimal places we estimate $\nu=4.426$ and so $\alpha=\nu^{1 / 3}=1.642$, which we remark differs from that at $\phi=0.5$ which in turn differs from that at $\phi=0$ [10], illustrating the varied scaling factors for different choices of $\phi$. As before we can substitute this solution into (3.11) to produce the $H$ functions, and then rescale them to produce $\tilde{H}$. Following this we plot the period three orbit of the renormalization operator $g_{n}(x, u)$ as shown in Figure 8 .
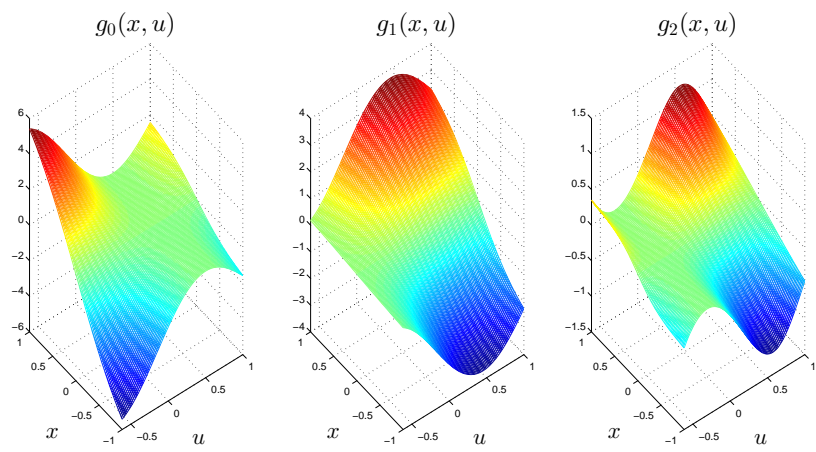

Figure 8: Plot of $g_{n}(x, u)$ showing the period three orbit of the renormalization operator.

We estimate the rate of decay of the amplitude $\mathcal{A}_{i}$ to follow a power law $i^{-\kappa}$ where $\kappa=\ln (\nu) / 3 \ln \left(\omega^{-1}\right)=1.030$, and this is shown in Figure 9a.

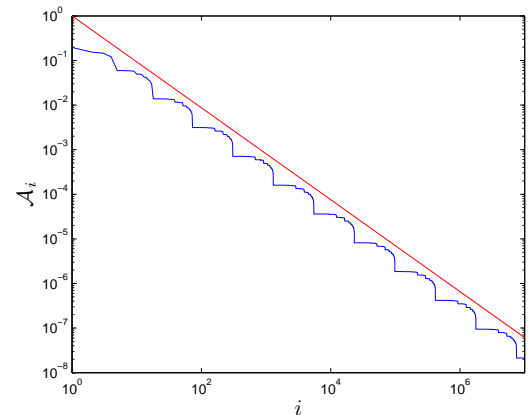

(a) Plot of $\mathcal{A}_{i}$ on a logarithmic scale, in addition to the decay rate $i^{-\kappa}$ suggested by the renormalization analysis

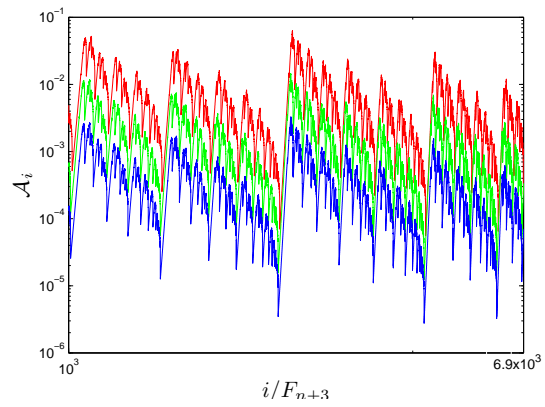

(b) Plot of the amplitude $\mathcal{A}_{i}$ against rescaled time for $\sigma=1+\omega^{n} \epsilon_{0}$ for $n=3,6$ and 9 (coloured red, green and blue respectively) where $\epsilon_{0}=0.1$. The vertical shift corresponds to the factor $\nu$.

Figure 9: Plots showing the derived scaling laws for the amplitude at and near the critical point.

Using the same argument as before, we conclude that the scaling factor for a perturbation from the critical point for $\sigma$ is $\omega^{-3}$. Thus a decrease in the perturbation by this factor will reduce the size of the attractor by the factor $\nu$, and with a rescaling in the neighbourhood of $\theta^{0}$ we will be able to reproduce the same picture at smaller and smaller scales. In Figure $9 \mathrm{~b}$ we show a plot of the amplitude $\mathcal{A}_{i}$ for $\sigma$ values near the $\mathrm{PCP}$ with time appropriately rescaled showing the self similar behaviour, and the vertical shift corresponds of course to the factor $\nu$. A similar plot was produced in [10] for the case $\phi=0$. Likewise we can produce the analogous plots to Figure 6 for the PCP point to show the self-similarity in the actual system, and this is shown in Figure 10. Note that these perturbations lie on bifurcation curve $C$ where we still have SNA, because the Lyapunov exponent on $\mathcal{L}$ is negative and the attractor 
crosses $\mathcal{L}$ on a dense set of points. Note that apart from $x$ being of fixed sign, the PCP behaves no differently than any point on the line $A$.

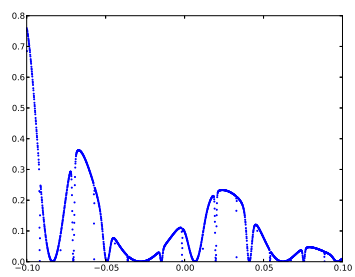

(a) SNA for parameter choice $(\phi, \sigma)=\left(1,1+\epsilon_{0}\right)$.

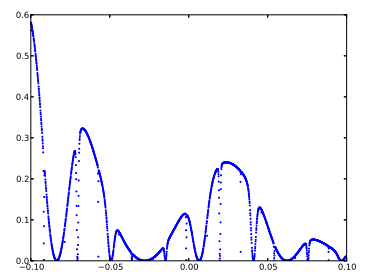

(c) Rescaled strange nonchaotic attractor for parameter choice $(\phi, \sigma)=\left(1,1+\omega^{6} \epsilon_{0}\right)$

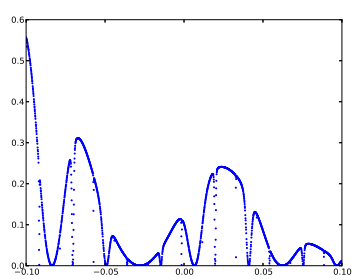

(b) Rescaled strange nonchaotic attractor for parameter choice $(\phi, \sigma)=\left(1,1+\omega^{3} \epsilon_{0}\right)$

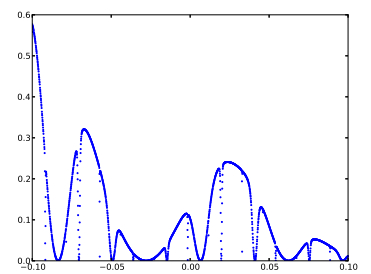

(d) Rescaled strange nonchaotic attractor for parameter choice $(\phi, \sigma)=\left(1,1+\omega^{9} \epsilon_{0}\right)$

Figure 10: Plots showing self-similarity near the critical point $(1,1)$ along bifurcation curve $C$ with $\epsilon_{0}=\delta_{0}=0.1$.

To finish this section we present tables for different initial phases and choices of $\phi$, which display the resulting period of $Q_{n}^{2}$ and the numerically calculated values of $\nu$ for these choices in Tables 1 and 2.

\begin{tabular}{|c|c|c|c|c|}
\hline$\phi$ & $\cos ^{-1}(-\phi) / \pi$ & Period of $Q_{n}^{2}$ & $\nu$ & $\alpha$ \\
\hline 0.1843469 & $1 / 4+\omega / 2$ & 12 & 90.07 & 1.455 \\
0.4665646 & $1 / 2+\omega / 4$ & 12 & 90.07 & 1.455 \\
0.5 & $2 / 3$ & 4 & 4.12674 & 1.42529 \\
$\sqrt{2} / 2$ & $3 / 4$ & 12 & 90.07 & 1.455 \\
0.8563191 & $3 / 4+\omega / 8$ & 24 & 90.07 & 1.206 \\
1 & $1 / 2$ & 3 & 4.426 & 1.642 \\
\hline
\end{tabular}

Table 1: Scaling factors obtained when $\theta^{0}=0$ is held constant and $\phi$ is varied

\section{Autocorrelation function}

We now focus purely on the sign of $x_{i}$, writing $s_{i}=\operatorname{sign}\left(x_{i}\right)$. The only way for $x$ to change sign is if the factor $\phi+\cos (2 \pi \theta)$ is negative. The zeros of this function are at

$$
\theta= \pm \frac{\cos ^{-1}(-\phi)}{2 \pi}+n, \quad n \in \mathbb{Z} .
$$




\begin{tabular}{|c|c|c|c|}
\hline$\theta^{0}$ & Period of $Q_{n}^{2}$ & $\nu$ & $\alpha$ \\
\hline 0 & 6 & 7.4246 & 1.3967 \\
$1 / 3$ & 24 & 3038 & 1.3967 \\
$1 / 8+3 \omega / 8$ & 12 & 93.7 & 1.4599 \\
$1 / 4+\omega / 4$ & 6 & 7.4246 & 1.3967 \\
$1 / 2+\omega / 4$ & 3 & 1.602820 & 1.170294 \\
$1 / 2+3 \omega / 4$ & 3 & 4.63223 & 1.66698 \\
\hline
\end{tabular}

(a) $\phi=0$

\begin{tabular}{|c|c|c|c|}
\hline$\theta^{0}$ & Period of $Q_{n}^{2}$ & $\nu$ & $\alpha$ \\
\hline 0 & 4 & 4.12674 & 1.42529 \\
$1 / 4$ & 12 & 12.12621 & 1.23115 \\
$1 / 2$ & 12 & 70.277 & 1.42529 \\
$w$ & 4 & 4.12674 & 1.42529 \\
$1 / 2+\omega / 4$ & 12 & 12.12621 & 1.23115 \\
$3 / 4$ & 12 & 407.3 & 1.650 \\
\hline
\end{tabular}

(b) $\phi=0.5$

\begin{tabular}{|c|c|c|c|}
\hline$\theta^{0}$ & Period of $Q_{n}^{2}$ & $\nu$ & $\alpha$ \\
\hline 0 & 3 & 4.426 & 1.642 \\
$1 / 4$ & 6 & 6.777 & 1.376 \\
$1 / 8+3 \omega / 8$ & 12 & 2832 & 1.939 \\
$1 / 4+\omega / 4$ & 6 & 6.777 & 1.376 \\
$1 / 2+\omega / 4$ & 6 & 6.777 & 1.376 \\
$3 / 4$ & 6 & 56.7 & 1.96 \\
\hline
\end{tabular}

(c) $\phi=1$

Table 2: Scaling factors obtained when $\phi$ is held constant and $\theta^{0}$ is varied

Following the approach in [11], the evolution of the $s_{i}$ can be written as the skew-product system

$$
\begin{aligned}
& s_{i+1}=\Phi\left(\theta_{i}\right) s_{i} \\
& \theta_{i+1}=\theta_{i}+\omega(\bmod 1),
\end{aligned}
$$

where

$$
\Phi(\theta)= \begin{cases}+1, & \theta \in[0, \alpha / 2] \cup[1-\alpha / 2,1] \\ -1 & \text { Otherwise. }\end{cases}
$$

Here we have let $\alpha=\cos ^{-1}(-\phi) / \pi$. This system can be "solved" and the solution is

$$
\begin{aligned}
& s_{n}=s_{0} \prod_{j=0}^{n-1} \Phi\left(\theta_{0}+j \omega\right), \\
& \theta_{n}=\theta_{0}+n \omega .
\end{aligned}
$$

If $\omega$ is rational then $s_{i}$ is periodic. However if $\omega$ is irrational this solution tells us nothing of the nature of the dynamics of $s_{i}$ and so in an effort to learn more we consider the autocorrelation function (ACF). The ACF at time $t$ is defined as

$$
C(t)=\lim _{N \rightarrow \infty} \frac{1}{n} \sum_{i=0}^{N} s_{n} s_{n+t} .
$$

This skew-product system is in fact the same as the symmetric barrier billiard system we have previously studied in [13]. Using a renormalization scheme, it is shown in [13] that (for the golden mean forcing frequency)

$$
C\left(F_{n}\right)=\frac{1}{(-\omega)^{-n}} \int_{0}^{(-\omega)^{-n}} Q_{n}(x) \mathrm{d} x
$$

where $Q_{n}$ is as defined in (3.10) but with initial conditions $Q_{0}=1$ and $Q_{1}(x)=\Phi(-\omega x)$.

Furthermore, it shown in [2] that if $\alpha \in \mathbb{Q}(\omega)$ then the ACF displays self-similarity at Fibonacci times (periodic behavior of $Q_{n}$ ). In the case $\alpha=1 / 2$ we asymptotically have peaks of magnitude $1-1 / \sqrt{5}$ at every third Fibonacci number, and the ACF is zero at all other Fibonacci times. This corresponds to the case of $\phi=0$ in our model, which reduces to qualitatively to the original SNA studied in [8]. A detailed analysis of periodic correlations for this system was performed in [11]. 
However in the Glendinning model we can vary the parameter $\alpha$, and hence the function $\Phi$. In symmetric barrier billiards this corresponds to varying the height of the barrier. It is important to note that for any analysis of signs to be valid we must take our parameter choice in Region 2 for which we have SNAs. This is because in Region 1 the attractor is quasiperiodic on the circle $x=0$, which has no sign and thus the ACF is undefined. In Region 3 the map becomes invertible and the sign of $x$ never changes, and so $C(t)=+1$.

In [13] we extended the analysis of [2] to include the general case of $\alpha \notin \mathbb{Q}(\omega)$. In this case we numerically approximated the time series in $\left(C\left(F_{n}\right)\right)$ and using Taken's embedding theorem [17] we embedded this series in three dimensional space, yielding a renormalization strange set as shown in Figure 11. Note that this set is produced independent of the choice of $\alpha$.

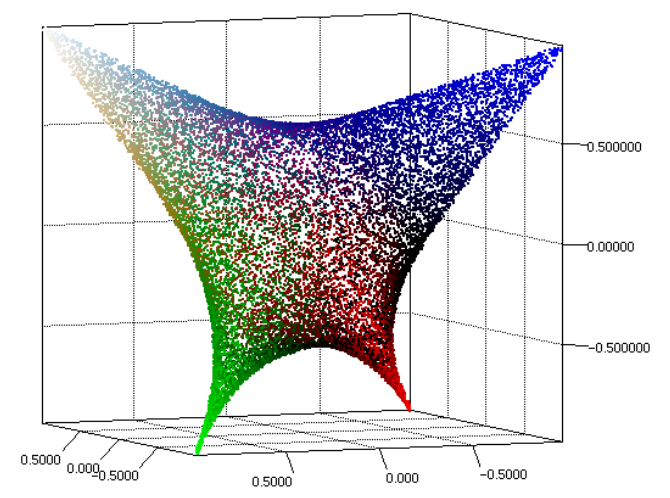

Figure 11: A plot of the triple $\left(C\left(F_{n}\right), C\left(F_{n+1}\right), C\left(F_{n+2}\right)\right)$, revealing an invariant set in three dimensional space on which the correlations lie.

The Lyapunov exponent on this set was numerically shown in [13] to be positive, thus indicating that the correlations are chaotic.

\section{Conclusion}

We have developed a renormalization group approach to describe the non-smooth pitchfork bifurcation initially studied in [7].

Following the approach in [10], we separated the dynamics into two component parts exhibiting different characteristics of the system under study. Of particular interest is recurrence (3.11) (which has not been broadly studied to this point) which is responsible for the main feature of interest - growth of the attractor. Assuming the growth of this sequence of functions is asymptotically constant over a period of forcing, we have shown that this recurrence suitably rescaled gives rise to a periodic orbit which can be used to construct the corresponding periodic orbit of the renormalization operator.

Another attempt at this problem in [5] (which featured a few minor errors which we have corrected in this paper) relied on the calculation of the scaling factor as the solution of an eigenvalue problem. However, we have found that the scaling factor can be estimated from the growth rate, which makes the numerics easier.

We have demonstrated that for certain choices of the parameter $\phi$ and initial phase $\theta^{0}$ the renormalization gives rise to self similarity of the system at and near the critical line $\sigma=1$. We provided a full analysis of the scaling at 
the PCP, which is of interest as it separates three regimes of dynamical behaviour.

Finally, we provided a link between the autocorrelation function seen in the study of symmetric barrier billiards [13] and that seen for the study of signs in the Glendinning model. We show that the two systems in this context are equivalent and that for a typical choice of parameter in Region 2 the correlations at Fibonacci times lie on a renormalization strange set which is the quasiperiodic equivalent to the so-called "orchid" set which occurs in the study of decaying eigenfunctions of the generalised Harper equation [12].

A generalization of this work to a class of quadratic irrational forcing frequencies should be straightforward. In [1] we extended our work on renormalization of the autocorrelation function in symmetric barrier billiards to such a class of frequency, and the renormalization equations for $Q_{n}$ for the Glendinning model will be identical to those in [1]. Indeed, the results from [1] can be directly ported over to the study of the autocorrelation function for the Glendinning model as shown in Section 4. Extending further to general irrational frequencies is more challenging as the renormalization equations change at every step, depending on the continued fraction expansion of the frequency.

\section{References}

[1] L N C Adamson and A H Osbaldestin, Renormalisation of correlations in a barrier billiard: Quadratic irrational trajectories, Physica D: Nonlinear Phenomena 270 (2014), 30-45.

[2] J R Chapman and A H Osbaldestin, Self-similar correlations in a barrier billiard, Physica D 180 (2003), no. 1, $71-91$.

[3] M. Ding, C. Grebogi, and E Ott, Evolution of attractors in quasiperiodically forced systems: From quasiperiodic to strange nonchaotic to chaotic, Physical Review A 39 (1989), no. 5, 2593.

[4] T. A Driscoll, N. Hale, and L. N. Trefethen, Chebfun guide, Pafnuty Publications, 2014.

[5] U Feudel, S P Kuznetsov, and A Pikovsky, Strange nonchaotic attractors: Dynamics between order and chaos in quasiperiodically forced systems, World Scientific, Singapore, 2006.

[6] U Feudel, A Pikovsky, and A Politi, Renormalization of correlations and spectra of a strange non-chaotic attractor, Journal of Physics A: Mathematical and General 29 (1996), no. 17, 5297-5311.

[7] P Glendinning, The nonsmooth pitchfork bifurcation, Discrete \& Continuous Dynamical Systems Series B 6 (2004), no. 4, 457-464.

[8] C. Grebogi, E. Ott, S. Pelikan, and J. A Yorke, Strange attractors that are not chaotic, Physica D 13 (1984), no. 1, 261-268.

[9] G Keller, A note on strange nonchaotic attractors, Fundamenta Mathematicae 151 (1996), no. 2, 139-148.

[10] S P Kuznetsov, A S Pikovsky, and U Feudel, Birth of a strange nonchaotic attractor: A renormalization group analysis, Physical Review E 51 (1995), no. 3, R1629-R1632.

[11] B D Mestel and A H Osbaldestin, Periodic orbits of renormalisation for the correlations of strange nonchaotic attractors., Mathematical Physics Electronic Journal [electronic only] 6 (2000), no. 5, 27.

[12] _ Golden mean renormalization for a generalized Harper equation: The Ketoja-Satija orchid, Journal of Mathematical Physics 45 (2004), 5042-5075.

[13] A H Osbaldestin and L N C Adamson, Chaotic correlations in barrier billiards with arbitrary barriers, Journal of Physics A: Mathematical and Theoretical 46 (2013), no. 24, 245101.

[14] A S Pikovsky and U Feudel, Correlations and spectra of strange nonchaotic attractors, Journal of Physics A: Mathematical and General 27 (1994), no. 15, 5209-5219.

[15] _ Characterizing strange nonchaotic attractors, Chaos: An Interdisciplinary Journal of Nonlinear Science 5 (1995), no. 1, 253-260. 
[16] F J Romeiras, A Bondeson, E Ott, T M Antonsen Jr, and C Grebogi, Quasiperiodic forcing and the observability of strange nonchaotic attractors, Physica Scripta 40 (1989), no. 3, 442.

[17] F Takens, Detecting strange attractors in turbulence (Lecture Notes Mathematics vol 898), Berlin: Springer (1981), 366-381. 